\title{
Una visita a Ricardo Rojas
}

\begin{abstract}
AlÅ por el mes de agosto de I954, estando en Buenos Aires, pasé A una tarde con Ricardo Rojas en su casa, hablándome de su labor y de sus ideas. Un resumen de lo que me dijo constituirá tal vez una documentación útil sobre el gran escritor argentino, quien por cierto en ese momento tenía la ilusión de conseguir el premio Nobel. Sabiendo que ese premio pocas veces se ha concedido con un amplio criterio intelectual, sospechaba de antemano que esa esperanza resultaría fallida.

Don Ricardo, a quien conocía desde hace muchos años, me recibió con su amabilidad habitual en su casa porteña en la calle de Charcas 2837, y después de atravesar el primer patio entramos en su despacho, donde nos sentamos a charlar. Hablamos primeto del gran caserón donde estábamos, y cuya arquitectura me hizo pensar en los edificios del Alto Perú colonial por la mezcla de arte española con detalles que, a pesar de ser europeos muchos de ellos en su origen, se cultivaton con especial frecuencia y con curioso sabor local en los edificos construidos por obreros indios. La casa es una casa colonial típica, con un patio delante y otro atrás, pero entre los adornos del primer patio se ven unas sirenas clásicas que, cosa rata, figuran con frecuencia hasta en las iglesias andinas. A esta arte mezclada se le ha dado el nombre de arte mestiza, pero Don Ricardo no empleó ni una vez esa palabra. Dijo que la casa era una manifestación artística de "Eurindia", y me explicó lo que entendia por ese neologismo inventado por él. Es una especie de fusión entre lo indio y lo europeo, teoría un poco mística que no se presta al análisis científico. Hay en ella reminiscencias de Keyserling, y Don Ricardo emplea la palabra "telúrico", alrededor de la cual se ha formado todo un complejo ideológico-sentimental en Suramérica. El concepto de Eurindia corresponde a la teoría de la raza cósmica que José Vasconcelos desarrolló en el curso de un viaje por la América austral. Es una teoría que no responde a las
\end{abstract}


preocupaciones sociológicas de la Argentina de hoy, más italianizada y metida francamente en el cauce del mundo occidental. Hay que recordar que don Ricardo nació en Tucumán, donde se siente todavía la influencia de la cultura mestiza del Alto Perú. Don Ricardo me informa que la fachada de sur casa bonaerense viene de Tucumán, pero insiste repetidamente que dicha casa no es colonial sino eurindia, siendo lo colonial, según él, la negación de lo eurindio. El arquitecto de la casa fue Angel Guido, ex-rector de la Universidad del Litoral y profesor de arquitectura en la misma Universidad, en Rosario. Ricardo Rojas me afirma que este gran amigo suyo es el arquitecto de Eurindia, de la misma manera que hay, según él, pintores y músicos de Eurindia. La casa se construyó hace un poco más de un cuarto de siglo, y es única en esa ciudad hoy totalmente afrancesada, europeizada y norteamericanizada en la arquitectura.

Hablando de su vida, don Ricardo afirma que no nació en Santiago del Estero, como lo creen muchos, sino en Tucumán, en I882, y que su fe de bautismo está en lo que es hoy la catedral de Tucumán (en aquella época era una matriz que dependía del obispado de Salta). Por el lado materno, Ricardo Rojas desciende de una muy antigua familia de Tucumán. Por el lado paterno, viene de una familia igualmente antigua de Santiago del Estero. Cuando don Ricardo nació, sus padres se habían establecido por unos años en Tucumán, pero cuando tenía apenas dos años volvieton a Santiago del Estero, que el padre de Ricardo, Absalón Rojas, representaba como diputado en el congreso nacional.

Don Ricardo habla con emoción y voz vibrante de la ciudad de Santiago del Estero, y confiesa que sus ideas derivan sin duda de la influencia subconsciente que en él tuvo esa tierra donde pasó su juventud. Insiste en la antigüedad de Santiago, que llama "la madre de las ciudades". Poco después de la conquista del imperio incaico por Pizarro, los españoles establecieron Santiago como un centro desde donde podrían conquistar lo que hoy es la Argentina, sometiendo a los indios y repartiendo sus tierras. Don Ricardo afirma que esto correspondía a un plan preconcebido, pero no explica por qué los españoles escogieron un lugar tan inhóspito, que él mismo compara con la Palestina. Tal vez haya contestado más tarde a esta duda mía, diciendo que antes había abundantes bosques en la región de Santiago, bosques que fueron talados sin piedad por generaciones posteriores. El primer español que intentó fundar la ciudad de Santiago fue don Diego de Rojas, pero fue muerto por los indios, y más tarde Francisco de Aguirre pudo establecerse alli con cincuenta hombres y unos mil indios amigos. Con la excepción de Cuyo, que fue colonizada desde 
Santiago de Chile, se puede decir que todas las ciudades argentinas derivan de Santiago del Estero. De ahí fueron fundadas las ciudades de Tucumán, Córdoba, La Rioja; Salta, Jujuy y muchas otras.

Don Ricardo fue un niño precoz. Gracias a su madre, Rosario Sosar aprendió a leer a los cuatro años. Hizo sus estudios en Santiago hasta la edad de I6 años, sacando su título de bachiller, y entonces fue a Buenos Aires, donde se matriculó en la Facultad de Derecho. Después de cuatro años tomó parte en una huelga de estudiantes, y jamás quiso volver a las aulas. Se declaró "estudiante libre y perpetuo", y sólo regresó más tarde cuando lo llamaron como profesor. Llegó a ser decano y rector sin haber sacado título alguno. Ya no era el derecho lo que le interesaba, sino las humanidades. Siendo rector de la Universidad de La Plata Joaquín V. González, don Ricardo fundó en esa institución en I909 la Facultad de Humanidades. Enseñaba literatura española, pero afirmaba la necesidad de crear cátedras de literatura nacional, es decir argentina, contra la oposición de los muchos que afirmaban que no había literatura argentina, ni siquiera hispanoamericana. Se hizo famoso don Ricardo con esta campaña a favor de la cultura nacional, y en 1925 fue elegido rector de la Universidad de Buenos Aires, regida en aquella época por la Ley Avellaneda, según la cual los rectores eran elegidos democráticamente. Más tarde se cambió este sistema, y los rectores fueron sencillamente nombrados por el gobierno nacional. Siendo rector por elección de I925 a 1929, don Ricardo gozó de un gran prestigio y de una gran libertad. Hasta llegó a expresar públicamente su desacuerdo con el Ministro de la Guerra, cosa siempre peligrosa en un país hispanoamericano. Quedó como profesor de la Universidad de Buenos Aires hasta 1946, cuando la dictadura de Perón lo puso fuera de la Universidad, así como a la mayoría de profesores de valor de las Universidades argentinas.

A pesar de la persecución de la cual era objeto, don Ricardo continuó actuando como maestro de la juventud. Su casa quedaba abierta a los estudiantes, quienes acudían libremente a consultarlo y a trabajar en su biblioteca. Don Ricardo había escrito mucho-sólo su Historiat de la literatura argentina tenia ocho tomos-y se dedicó ya a organizar sus trabajos para la edición completa de sus obras en unos 40 tomos que había empezado a publicar la casa editorial Losada.

Don Ricardo se había entregado a su obra literaria, sin pensar en la política o por lo menos sin afiliarse a ningún partido, hasta el golpe de 6 de septiembre de I930, cuando los militares, inspirados por el ejemplo italiano, derrocaron al gobierno y echaron de lado la vida institucional, 
sin hacer el menor caso de la Constitución de 1853 , que se había convertido para los argentinos cultos en una cosa sagrada, como, por ejemplo, la Constitución norteamericana o la española de I8I2. Viendo desairado al Partido Radical, que quería que se respetara la Constitución, don Ricardo no vio otra solución que afiliarse al Partido perseguido. Inmediatamente el Partido Radical, conmovido por el gesto muy noble de don Ricardo, lo nombró secretario general, y después presidente de la comisión política y presidente de la convención nacional. Se presentó como candidato a diputado, pero el gobierno desautorizó su candidatura, así como las de Alvear y Güemes. Le pasó algo semejante cuando quiso presentarse como candidato al Senado. Hasta en los momentos más dificiles, don Ricardo se mostró fiel a los ideales constitucionales.

Después de charlar de su vida política, don Ricardo y yo pasamos en revista su obra literaria. La primera obra que publicó fue un poemá titulado La victoria del bombre (1903), poema en el cual exalta el triunfo del hombre sobre su medio físico y el comienzo del hombre como ser superior. Pero don Ricardo fue siempre más bien prosista que poeta, y escribía artículos sobre una gran variedad de temas en distintos diarios de la capital argentina. Algunos de estos artículos fueron reunidos en el libro que salió en I904, bajo el título de Cosmópoliss. Escribía además artículos sobre su especialidad, es decir, la literatura española. Algunos de sus trabajos fueron reunidos y publicados bajo el título de El alma española (I905). Todavía no había visitado a España. Las impresiones del viaje que hizo posteriormente a ese país se encuentran en el libro El retablo español. Su interpretación de España coincidía con la de su amigo Miguel de Unamuno. Veía la tragedia de un pueblo que, a pesar de sus valores humanos, no consigue ordenar su vida política. Don Ricardo atribuye eso al hecho de que España haya sido gobernada por dinastías extranjeras, primero los Austrias y después los Borbones, teoría que no puede aceptar el que escribe, ya que siempre es una solución fácil, passing the buck, como se dice en inglés, el atribuir las desdichas de un país a los extranjeros, teoría netamente nacionalista.

En 1906, Rojas publicó una serie de cuentos bajo el título de $E l$ Ucumar y La psiquina. El primero de estos nombres raros deriva de una leyenda de Santiago del Estero, y se refiere a un ser monstruoso, casi inhumano. La psiquina es el nombre que Rojas dio a un alcaloide como el del opio descubierto por aquella época, pero que produce efectos más sutiles que el opio. Esta obra, poco leída hoy, porque los oprum-eaters no están de moda, tuvo bastante éxito, y fue traducida al inglés por el 
norteamericano Peter Goldsmith, bajo el titulo The Mysterious Alcaloid. Hablando de su obra El país de la selva (1907), don Ricardo se acuerda con tristeza y hasta con amargura de la transformación que ha sufrido la región de Santiago del Estero como consecuencia de la introducción de la vida comercial moderna. Habla con añoranza del algarrobo, que tanto significaba para el habitante primitivo de la región, y me cita sus versos "La cosecha del algarrobo". Los quebrachos fueron derribados para hacer durmientes para los ferrocarriles.

El nacionalismo telúrico de Rojas no le impedía buscar las vinculaciones de la Argentina con el mundo exterior; en esto también se parece a Vasconcelos. Estudió con mucha simpatía los países limítrofes de la Argentina, siendo recibido con gran cariño en el Brasil, país donde hay en general poca simpatía por la cultura argentina. Viajó por España, y en Crónicas de Francia, Inglaterra e Italia (r908), describe sus andanzas por aquellos paises. Le interesaba la historia universal, pero siempre vista desde el ángulo argentino.

En I908 Rojas publicó una obra difícil de juzgar: La restauración nacionalista. El uso de la palabra nacionalista siempre es peligroso, pero en aquella época no tenía el sentido peyorativo que tiene hoy, y parece que en la Argentina no designaba todavía a un determinado grupo politico, con el cual es poco probable que Rojas se haya identificado. $L A$ restauración nacionalista es una crítica del sistema educativo de la Argentina, que él ve como una imposición extranjera que no se adviene al ambiente argentino. Rojas ridiculiza el sistema burocrático, verbalista, teórico, empleado en la instrucción pública argentina. Parece una crítica de la influencia francesa y española en la instrucción argentina. Rojas habla con simpatía de los métodos norteamericanos, pero tampoco quiere que la Argentina imite los Estados Unidos. Dice que la instrucción argentina debe adaptarse al ambiente argentino, pero nunca explica en qué consiste exactamente eso, ya que hay muchos ambientes argentinos. Esta actitud refleja el nacionalismo inconsciente pero muy fuerte de algunos intelectuales hispanoamericanos. Lo puedo comparar con la pregunta que un intelectual muy fino le hizo a un ingeniero guatemalteco, y que no dejó de sorprenderme: "¿Han encontrado Uds. una manera guatemalteca de construir carreteras?" Aunque es difícil decir exactamente qué es lo que preconiza Rojas, no hay duda que se opone a la instrucción puramente académica y europeizante que predominaba en la Argentina. Antes de Rojas, los pedagogos argentinos no habían formulado las preguntas fundamentales sobre el sistema de enseñanza utilizado en la Argentina. 
La interpretación "nacionalista" de la historia argentina por Ricardo Rojas se ve en su amena natración Blasón de plata (rgro), en la cual cuenta, como en un romance en prosa, el desarrollo de la Argentina (país de plata) desde la época de los indios. Describe las distintas tribus, su religión, y la variedad lingüistica que representaban las tres grandes lenguas (quechua, guaraní, araucano) y los cincuenta y tantos dialectos. Cuando vino el hombre blanco hubo mestización, $y$, aunque el argentino de hoy es mucho más blanco que en el momento de la independencia, Rojas cree que hay un tipo físico argentino, una manera de hablar argentina, y hasta una psicología argentina. Tal vez sin darse cuenta, don Ricardo se estaba metiendo en ese asunto tan discutido por los sociólogos de hoy sobre las características nacionales. Los sociólogos que niegan la existencia de los tipos nacionales, y son muchos, estarían en total desacuerdo con las ideas de don Ricardo. Esto no quiere decir que dichos sociólogos tengan razón. Por lo general, están buscando leyes universales, y parece que les molesta la posibilidad de que haya diferencias nacionales fundamentales. En la época colonial, los argentinos vivían espiritualmente en el culto al rey y a la Iglesia Católica. Ese mito fue reemplazado con la independencia por el patriotismo que unía a los patricios y a los gauchos, a pesar de la distinción de su estado social. Parece que don Ricardo no previó o no dio importancia a la lucha entre la oligarquía y los descamisados.

La obra más voluminosa de Rojas es su Historia de la literatura argentina, cuyos cuatro tomos de la edición primitiva (más tarde llegaron a ser ocho) corresponden a los cuatro temas que constituyen la división fundamental de la obra. El primer tomo se titula Los gauchescos, y en él Rojas ha reunido todo lo que tenga relación con la literatura popular, no sólo del campo sino de la ciudad. Con su culto de lo telúrico y de lo eurindio, es natural que Rojas haya tratado primero este tema para darle una base popular a la literatura más culta. El segundo tomo, Los coloniales, describe los tres siglos de historia colonial e insiste en la evangelización del país, así como en el fenómeno que le acompaña, el triunfo lento del castellano sobre los dialectos indígenas. En la época colonial se formaron instituciones como el cabildo, que tanta importancia habían de tener en la historia posterior del país. Después de la independencia en I8ıo vinieron las guerras civiles, que obligaron a muchos de los espíritus más finos a refugiarse en otros países. Sarmiento escribió su Facundo en el destierro, Alberdi sus Bases, y Echeverría sus obras doctrinarias. Es por esto por lo que Rojas puso el título de Los proscriptos al tercer tomo, que describe la literatura argentina del siglo XIX. La época moderna ha traído a la Ar- 
gentina una vida tan variada que Rojas no encontró para el cuarto y último tomo otro título que Los modernos.

Los que quieren conocer la filosofía racial y telúrica de don Ricardo a la cual hicimos alusión antes deberían estudiar la obra Eurindia, que salió en r922. Como estas obras teóricas son siempre las que sobreviven menos, Eurindia está casi olvidada hoy, aunque en su época tuvo bastante repercusión.

Hombre de múltiples facetas, don Ricardo se ha dedicado también al teatro. En I929 estrenó un drama en verso Elelin, que tiene como fondo la entrada de Diego de Rojas en el norte de lo que debía ser la Argentina para establecer el pueblo de Santiago del Estero. Como dijimos antes, Diego de Rojas fue asesinado, peto don Ricardo utiliza ese episodio para describir el impacto de los dos pueblos que debían crear lo que él llama lo "eurindio" o "lo criollo". Su segundo drama La casa colonial, estrenado en 1932 con Eva Franco como primera actriz, también tiene un fondo histórico. Esta vez estamos en el año I8I2, año de la conspiración de Alzaga que tenía como fin derrocar al nuevo gobierno patriótico. En su primer drama, don Ricardo describe la llegada de los españoles, en éste el fin del régimen español: sobre un fondo de pasión patriótica provocada por la lucha entre padres españoles e hijos criollos, se desarrolla una historia de amor, contraste que con algunas diferencias ha sido el tema de innumerables dramas y novelas en el mundo entero. Poco después del estreno de La casa colonial, se dio en el Teatro Cervantes, que es el teatro nacional de la Argentina, el drama de Rojas, Ollantay, basado en el supuesto drama quechua del mismo nombre, y que, claro está, nos lleva de nuevo a los albores de la historia de Eurindia. Entre los dramas de Rojas merece mención también la obra La salamanca, que cuenta una leyenda de magia y de brujería que atravesó el Atlántico con los españoles. Detiva de la conocida superstición según la cual en la ciudad universitaria de Salamanca, bajo la iglesia de San Ciprián, había una especie de universidad diabólica. Don Ricardo dice que este drama que describe al Diablo como un profesor de ciencias ocultas es una especie de Fausto americano.

Tal vez sea el Ricardo Rojas biógrafo el que ha tenido más éxito, con sus vidas de San Martín y de Sarmiento. Su biografía del libertador, que salió en 1933 bajo el título El santo de la espada, ha vendido más de 200,000 ejemplares. Dice el refrán inglés, "Nothing succeeds like success", pero también se puede decir que no hay nada como el éxito para despertar envidias y críticas. Se podría haber criticado al libro de Rojas 
por haber abusado de la palabra "santo", que al fin y al cabo es una palabra sagrada, y San Martín habrá sido muchas cosas pero santo no era. Sin embargo, que yo sepa, no se hizo esa crítica, mientras que los argentinos denunciaron con violencia el sacrilegio que representaba el deseo de Perón de dar a Evita el título de "santa" (aunque, como decimos Don Quijote y yo, las comparaciones son siempre odiosas). Los ataques contra el libro El santo de la espada vinieron de distintos lados. Don Ricardo había querido escribir una biografía popular de San Martín, que, siendo rigurosamente histórica, no llevara notas de pie de página y estuviera escrita en un estilo ameno. Los historiadores profesionales, con esa psicologia de perro del hortelano que los caracteriza, pusieron el grito en el cielo, como lo hicieron por ejemplo contra Salvador de Madariaga. Para contestar a sus detractores, don Ricardo publicó un folleto titulado Una lección de bistoria, en el cual afirma que cada detalle de su biografía está bien documentado. También se molestaron los nacionalistas militares (que no hay que confundir con los nacionalistas a lo Ricardo Rojas). El grupo representado por Perón quiso hacer de San Martín su santo patrono, y Perón creó un culto oficial de San Martín, quien vendría a ser una especie de descamisado con uniforme. A este grupo les molestaba profundamente que la biografía más popular sobre San Martín, y al fin y al cabo la mejor escrita, fuera obra de un intelectual, de un radical, de un individualista que se habia metido en la política porque le repugnaba el uso que hacían las espadas de la espada de San Martín, de un constitucionalista que despreciaba a Rosas (a quien los historiadores revisionistas estaban ensalzando), y que al contrario escribió otra gran biografía de Sarmiento para probar que la auténtica tradición argentina venía de San Martín por Sarmiento a los liberales de hoy, y no de San Martín a través de Rosas a Perón y a sus secuaces. El grupo peronista hizo todo cuanto pudo para impedir la venta de esta obra de Rojas. Otro grupo que se indignó contra don Ricardo fueron los venezolanos, y de manera especial Vicente Lecuna, quien, con una ausencia total de sentido filosófico, se molestaba profundamente si alguien no adoptaba una actitud de reverencia religiosa delante de la figura de Simón Bolívar, ya que él no permitía más que cantos y loores ad majorem dei gloriam. Ha habido una rivalidad inevitable entre Bolívar y San Martín para la primacía histórica. Vicente Lecuna no se entusiasmó con el éxito de la biografía de San Martín, y se puso netamente receloso cuando en 1947 don Ricardo publicó La entrevista de Guayaquil, donde se desarrollaba el tema ya indicado en El santo de la espada, es decir que, en esa famosa entrevista, San Martín mostró una actitud noble 
y que no se sentía humillado delante del genio supetior de Bolívar, como lo han pretendido algunos historiadores venezolanos. Lecuna no se pudo contener cuando poco después salió San Martín y el Congreso de Panamá, obra en la cual don Ricardo quiso explicar las razones por las cuales San Martín y el gobierno de Buenos Aires se decidieron a no tomar parte en dicho congreso. Lecuna denunció públicamente a Ricardo Rojas, cosa que éste naturalmente lamentó muchísimo.

Después del éxito del libro sobre San Martín, don Ricardo se dedicó con entusiasmo y con verdadera devoción al estudio de Sarmiento. Era muy amigo del nieto de Sarmiento, Augusto Belin Sarmiento (ya fallecido), quien llamaba a Rojas "el verdadero nieto de Sarmiento". Don Ricardo estudió con ahinco los cincuenta y dos tomos de las Obras Completas de Sarmiento que publicó este amigo, el nieto efectivo, carnal del gran enemigo de Rosas. En el curso de los años, don Ricardo publicó una serie de trabajos menores sobre Sarmiento, como, por ejemplo, sus libros La condición del extranjero en América y El pensamiento vivo de Sarmiento. Todos estos libros, monografías y artículos no eran más que un prólogo para la gran obra El profeta de la pampa, que salió en I945, cuando el debate sobre Sarmiento había llegado a su punto álgido y cuando historiadores nacionalistas como Diego Molinari lo estaban atacando constantemente. Claro está que el libro no gustó a este grupo, aunque por otro lado la Sociedad Argentina de Escritores le concedió su gran premio de honor. Nótese que de nuevo Rojas da a su héroe un título religioso. Tal vez haya en el uso de la palabra "Profeta" una cierta malicia, como para decir que Sarmiento preveía el verdadero porvenir de la Argentina, a pesar del éxito transitorio que puedan haber tenido sus detractores alrededor del año en que salió esta biografía.

Don Ricardo ya conocía la persecución política, como se ve en dos obritas que tienen bastante importancia personal porque don Ricardo no puede hablar de ellas sin emoción. Después de la publicación de El profeta de la pampa, en 1946, dio a conocer una obra que había escrito mucho antes, en I933, cuando otra dictadura lo persiguió. Se trata de El albatros, canto cívico en tercetos, que don Ricardo escribió en la prisión austral de Ushuaía. Escogió la forma dantesca del terceto para indicar que él se sentía como un Dante argentino, desterrado e indignado por los abusos de un gobierno corrompido. Un día, estando él en Santa Fe, lo tomó preso la policía, lo llevó a la isla de Martín García, y de allí un buque de guerra lo condujo a Ushuaía. No supo nunca de qué se le acusaba, no hubo proceso ni denuncia ni nada, aunque en otra ocasión don Ricardo 
fue procesado por "desacato", término que emplean muchos dictadores hispanoamericanos para disfrazar el abuso de la fuerza, como observa con justicia la víctima. Todo esto se pudo hacer porque estaba la Argentina en estado de sitio, y don Ricardo se vuelve elocuente también cuando denuncia este otro pretexto que utilizan los caudillos hispanoamericanos para maltratat a la población. Una noche, estando don Ricardo en Ushuaía, los esbirros del gobierno allanaron su casa, sin que su pobre mujer se los pudiera impedir. Por fin, sin explicación alguna, la policía llevó a don Ricardo a Buenos Aires, y sin una palabra, lo dejó de noche en una calle solitaria. Otra obra que don Ricardo escribió en Ushuaía fue un tratado en prosa titulado Archipiélago, en el cual Rojas describe esa región casi desconocida de la Argentina austral, y pregunta por qué el gobierno no hace nada por desarrollarla.

Después del periodo peronista, Ricardo Rojas tenia el derecho bien ganado de descansar, pero sigue trabajando, poniendo un capítulo final a su obra. Tal vez ya hayan salido a luz tres obras autobiográficas que serán muy útiles para los que quieran estudiar la vida de este notable argentino. La primera es la biografía de su padre, que tomó una parte activa en la vida nacional; la segunda una descripción de la infancia y juventud de don Ricardo en Santiago del Estero; la tercera, titulada Entre bobemios y doctores, cuenta su llegada a Buenos Aires como estudiante, y nos da una visión de cómo era la capital argentina en aquella lejana época.

Así terminó la larguísima conversación que tuve con don Ricardo. Ya conocía su vasta obra, y también lo conocía a él, pero quería hacerle una serie de preguntas sobre su vida y sobre sus libros. Es probable que este artículo no traiga al lector el calor humano de la entrevista. En este mundo mecanizado donde los profesores y hasta los escritores van a congresos aburridos para leer trabajos insignificantes en voz monótona, sin que nadie se atreva a decir que todo eso es una parodia de la vida intelectual, es un placer visitar una casa amena (en vez de un despacho universitario) y poder charlar con un conversador sumamente fino, cortés, bien informado y que sabe hablar. Este era uno de los aspectos esenciales de la civilización occidental. Me temo que, cuando vuelva a Buenos Aires, conozca una vida académica más "moderna", y que tenga que conversar con profesores-empleados (como muchos que conozco en Estados Unidos), y que nuestra conversación se parezca a la visita de un agente viajero a un pequeño comerciante para venderle una caja de jabón. Tal vez sea justo que le hayan torcido el cuello al cisne (creyendo que era papagayo), pero, ¿por qué cree este mundo progresista que es progreso cortar los hilos 
de la conversación culta, y que la vida culta no tiene nada que ver con la vida universitaria? Seamos, pues, fieles no sólo a don Ricardo Rojas, sino también y sobre todo a la manera de vida que él tan dignamente representa.

\author{
RoNALd HiLTON, \\ Universidad de Stanford.
}


